\title{
Caracterización del proceso de tutoría psicopedagógica en la Universidad Técnica Estatal de Quevedo, desde el Modelo Educativo por Competencias
}

\section{Characterization of the process of psychopedagogical tutoring in the State Technical University of Quevedo, from the Educational Model by Competencies}

\author{
Pablo Parra Silva ${ }^{1}$ \\ pparra@uteq.edu.ec \\ Badie Cerezo Segovia ${ }^{1}$ \\ bcerezo@uteq.edu.ec \\ Daniel Parra Gavilanes ${ }^{1}$ \\ dparra@uteq.edu.ec \\ Mirella Flores Jaén ${ }^{1}$ \\ mflores@uteq.edu.ec
}

Recibido: 1/07/2018, Aceptado: 1/09/2018

\begin{abstract}
RESUMEN
Con la identificación del estado actual de las tutorías psicopedagógicas en la Universidad Técnica Estatal de Quevedo (UTEQ), se busca determinar el desarrollo logrado con el Modelo Educativo por Competencias (MPC), cuya aplicación se inicia en el año 2008. El objeto de investigación es el proceso de tutoría psicopedagógica de la UTEQ, diez años después de la aplicación de su diseño. El objetivo es señalar la caracterización actual de este proceso de tutorías psicopedagógicas, con un modelo educativo sobre el cual se fundamentan las acciones educativas y construcción profesional. Este modelo determina las características del proceso de tutorías psicopedagógicas. Por lo que se deberán conocer las dificultades y los aciertos, en la situación actual de la institución educativa, desde la perspectiva de los/las estudiantes.
\end{abstract}

Palabras clave: Proceso de tutoría psicopedagógica, modelo educativo por competencias, construcción profesional

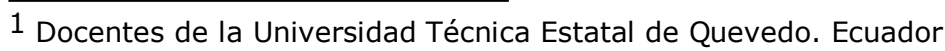




\begin{abstract}
With the identification of the current state of the psychopedagogical tutorials in the State Technical University of Quevedo (UTEQ), it is sought to determine the development achieved with the Educational Model by Competencies (MPC), whose application begins in the year 2008. The object of investigation is the process of psychopedagogical tutoring of the UTEQ, ten years after the application of its design. The objective is to point out the current characterization of this process of psychopedagogical tutoring, with an educational model on which the educational actions and professional construction are based. This model determines the characteristics of the process of psychopedagogical tutoring. So you should know the difficulties and the successes, in the current situation of the educational institution, from the perspective of the / the students.
\end{abstract}

Keywords: process of psychopedagogical tutoring, educational model by competencies, professional construction

\title{
Introducción
}

El proceso de tutoría psicopedagógica en la Universidad Técnica Estatal de Quevedo (UTEQ), en aplicación del Modelo Pedagógico por Competencias (MPC), tuvo su origen en el año 2008 y a la presente, muestra un conjunto de inconsistencias técnico-metodológicas, didácticas, axiológicas, de compromiso institucional, de capacitación y actualización, que de manera directa afectan los resultados de desarrollo integral que el docente-tutor debe asumir como su responsabilidad laboral.

El objetivo de la presente investigación es describir la caracterización actual del proceso de tutorías psicopedagógicas, con un modelo educativo sobre el cual se fundamentan las acciones educativas y construcción profesional. Lo cual está en línea con los nuevos roles de las instituciones de educación superior, particularmente en América Latina (Horruitiner, 2010; González Maura, 2006).

Los documentos de la institución de educación superior

El Modelo Pedagógico por Competencias aprobado en el 2008 y ratificado en el 2010, señala que "el trabajo metodológico es la labor que, apoyado en la didáctica, realizan los actores que intervienen en el proceso de enseñanza aprendizaje, con el propósito de alcanzar óptimos resultados, para satisfacer plenamente las competencias profesionales y los objetivos formulados en los planes de estudio" (UTEQ, 2012).

"El trabajo metodológico se concreta, fundamentalmente, en el desarrollo con calidad del proceso de enseñanza-aprendizaje, logrando una adecuada integración de las actividades como: clases, consultas y tutorías, con el trabajo autónomo del estudiante, la actividad investigativa y laboral; además con las tareas de alto impacto social y otras de carácter extracurricular que cumplen los estudiantes. Su esencia radica en la preparación de los académicos" (UTEQ, 2012). Así dice, expresamente este Artículo: la preparación de los académicos.

En cuanto al trabajo metodológico "es la auto-preparación que realiza el académico en los aspectos científico, técnico, didáctico, filosófico, político ideológico e informáticos requeridos para el desarrollo de su labor docente. Se ejecuta tanto de 
forma individual como colectiva. La capacitación de los académicos y tutores es una prioridad de esta labor" (UTEQ, 2012). La aplicación del Modelo por Competencias no está acabada. Por el contrario, su fundamento es la capacitación y actualización, que permita el fortalecimiento de todos los indicadores señalados, con igual prioridad y exigencia.

"El académico de la Unidad de Aprendizaje es aquel que maneja el nivel organizativo base y desempeña un papel fundamental en la formación integral del estudiante. Es el responsable fundamental de que esta (sic) sea impartida con la calidad requerida, desarrollando una labor educativa desde la instrucción. Para ello debe poseer una adecuada preparación pedagógica y dominar los contenidos fundamentales de la ciencia que explica; así como, orientar, controlar y evaluar a los estudiantes para lograr un adecuado dominio de dichos contenidos, en correspondencia con las competencias, los objetivos generales del módulo y de la unidad de aprendizaje, contribuyendo así a su formación integral" (UTEQ, 2012).

Los académicos del módulo, tendrán como principales funciones, según el Artículo 9 del Reglamento: a) Lograr el mejor desarrollo del proceso de enseñanzaaprendizaje garantizando el cumplimiento del programa general del módulo; b) La actualización permanente de los contenidos de las unidades de aprendizaje; c) Un enfoque metodológico adecuado para su desarrollo, teniendo en cuenta las relaciones horizontales y verticales que se establecen en el plan de estudio de la carrera; d) La participación en el diseño de los planes de estudio; e) La participación en el proceso de diagnóstico y evaluación integrales (sic) de los estudiantes; f) La conducción y el control sistemático de la marcha del proceso de enseñanzaaprendizaje desarrollando acciones para eliminar las deficiencias detectadas y proponiendo las medidas que permitan el mejoramiento continuo de la calidad de dicho proceso; g) La organización, planificación, ejecución, control y evaluación de las tareas de sus unidades de aprendizaje que contribuyan al proyecto integrador" (UTEQ, 2012).

Las formas y tipos del trabajo metodológico son: docente-metodológico y científicometodológico. El Artículo 16 del MPC, señala que "el trabajo docente- metodológico es la actividad que se realiza con el fin de mejorar de forma continua el proceso de enseñanza-aprendizaje; basándose fundamentalmente en la preparación didáctica que poseen los académicos, así como en la experiencia acumulada". Por su parte, el trabajo científico-metodológico, lo indica el Artículo 17, como "la actividad que realizan los académicos en el campo de la didáctica, con el fin de perfeccionar el proceso de enseñanza-aprendizaje, desarrollando investigaciones, o utilizando los resultados de investigaciones realizadas, que tributen a la formación integral de los futuros profesionales. Los resultados del trabajo científico-metodológico constituyen una de las fuentes principales que le permiten al académico el mejor desarrollo del trabajo docente- metodológico" (UTEQ, 2012).

El Artículo 18 del Reglamento, sostiene que los tipos fundamentales del trabajo académico-metodológico son:

a) Auto-preparación del académico;

b) Preparación de la unidad de aprendizaje;

c) Reunión metodológica;

d) Clase metodológica; 
e) Clase abierta; y,

f) Taller metodológico.

La auto-preparación del académico es "el tipo de trabajo docente-metodológico dirigido a asegurar su adecuada actualización y nivel científico-técnico y pedagógico con el fin de desarrollar el proceso de enseñanza-aprendizaje con la calidad requerida" (UTEQ, 2012). El Trabajo Académico "es la ejecución del proceso de enseñanza aprendizaje dirigido a lograr el cumplimiento de las competencias profesionales y los objetivos generales que se establecen en los planes y programas de estudios de las carreras". "En el trabajo académico - señalado en el Artículo 61se debe tener en cuenta la formación de principios y valores que se concreta en todas las actividades que se realicen. De esta forma, se propicia la formación integral de los estudiantes, desde unidades de aprendizaje y los módulos previstas en el plan de estudio. Este enfoque se debe adecuar a las particularidades del modelo pedagógico y a las características bajo las cuales se desarrolla el aprendizaje de los estudiantes, a los cuales se les debe brindar una atención personalizada" (UTEQ, 2012).

El Artículo 62 del Reglamento del MPC, fortalece los criterios que se mantienen en esta investigación, esto es que "la forma organizativa del trabajo académico es la estructuración de la actividad del académico y de los estudiantes, con el fin de lograr de la manera más eficiente y eficaz el cumplimiento de las competencias profesionales y de los objetivos previstos en los planes y programas de estudio. En el desarrollo de las diferentes formas organizativas es esencial que el académico garantice la actividad y la comunicación de los estudiantes en un clima afectivo y logre despertar el interés por el contenido- objeto de aprendizaje, de modo que se sientan comprometidos con su propio proceso formativo" (UTEQ, 2012).

Las formas organizativas del proceso enseñanza-aprendizaje, de acuerdo al Artículo 63, son: a) La clase; b) Las prácticas o pasantías pre profesionales; c) La vinculación con la colectividad; d) El trabajo investigativo de los estudiantes; e) La auto preparación de los estudiantes; f) La consulta; y, g) La tutoría. Estas formas organizativas se desarrollan en diferentes escenarios educativos. Se pueden incluir otras formas organizativas en aquellas carreras cuyas particularidades lo justifiquen, las que deben ser previstas en el plan de estudio" (UTEQ, 2012). Destáquese el papel de la tutoría como una forma organizativa del proceso de enseñanza-aprendizaje.

Importancia pedagógica revela el Artículo 66 del Reglamento del MPC, señalando que "la conferencia es el tipo de clases que tiene como objetivo principal la transmisión a los estudiantes de los fundamentos científico-técnicos más actualizados de una rama del saber mediante el uso adecuado de métodos científicos y pedagógicos, de modo que les ayude a la integración de los conocimientos adquiridos y en el desarrollo de las habilidades y valores que deberá aplicar en su vida profesional" (UTEQ, 2012).

Los tipos fundamentales del trabajo investigativo de los estudiantes son: el proyecto integrador del módulo y el trabajo de graduación y titulación. El proyecto integrador del módulo es el tipo de trabajo investigativo de los estudiantes "que les permite, mediante la solución de problemas profesionales, profundizar, ampliar, 
consolidar y generalizar los conocimientos adquiridos; aplicar, con independencia y creatividad, las técnicas y los métodos en otras formas organizativas del proceso de enseñanza aprendizaje y desarrollar los métodos del trabajo científico", como lo señala el Artículo 77 (UTEQ, 2012).

Es en el Artículo 86 del Reglamento del MPC, donde se especifica que "la tutoría es la forma organizativa que tiene como objetivo específico asesorar y guiar al estudiante durante sus estudios, para contribuir a su formación integral, realizando sistemáticamente acciones educativas personalizadas. Esta atención se realizará con encuentros, planificados por el profesor tutor, así como encuentros adicionales de acuerdo con las necesidades de los estudiantes y de los tutores. Deberá lograrse que el tutor sea el mismo desde el inicio de la carrera hasta que concluya sus estudios. El contenido de la tutoría estará dirigido esencialmente a la orientación y respuesta a las principales necesidades de los estudiantes, identificadas en su diagnóstico. Entre sus principales tareas están:

a) Diagnosticar al estudiante (dimensión socio familiar y académica);

b) Elaborar un plan de acción individual y/o grupal;

c) Elaborar y aplicar acciones educativas grupal e individual" (UTEQ, 2012).

El Artículo 87, señala que "el académico tutor desempeña un papel esencial en la formación integral del estudiante y tiene la responsabilidad de integral el sistema de influencias educativas presentes en los distintos ámbitos de su desarrollo personal. Para ello, debe poseer una preparación pedagógica que le permita identificar las necesidades educativas de los estudiantes, realizar las acciones personalizadas que correspondan y valorar la efectividad de las mismas" (UTEQ, 2012).

El modelo educativo y el proceso tutorial psicopedagógico en la UTEQ

La investigación buscará conocer lo que piensan los/las universitarios/as respecto a sí mismos, a sus docentes-tutores, a sus tutorías, a los planes de acción tutorial y en consecuencia también, a sus resultados en cuanto a su formación integral.

"Se señala que el reconocimiento del carácter activo y central del estudiante en el proceso educativo conlleva a la incorporación de un modelo pedagógico no tradicional, con el que se debe propiciar el intercambio entre los diversos actores en la construcción conjunta del conocimiento. Ello estimula una formación más completa en los educandos al concientizar el carácter inacabado y sistemático del saber. Al mismo tiempo, se requiere de una preparación más sólida del docente para que pueda fungir como orientador del proceso" (Travieso y Figuerola, 2014, pág. 29).

"Se reconoce la distancia que separa al currículo pensado, en tanto proyecto, de su aplicación práctica. Y es que, durante la actividad real, suceden situaciones inesperadas para el profesor, quien debe enfrentarse a dinámicas de difícil manejo pero necesarias, las cuales requieren de gran preparación pedagógica. El conocimiento de las regularidades psicológicas del joven universitario puede contribuir de manera positiva a esto, debido a que permite comprender las causas de muchos fenómenos que ocurren en el aula y prever sus futuras manifestaciones" (Travieso y Figuerola, 2014, pág. 29). 
Humarán y Domínguez, citan a Domínguez e Ibarra, entendiendo que "la proyección futura de la personalidad ha sido entendida y definida como la estructuración e integración de un conjunto de motivos elaborados en una perspectiva temporal futura, a mediano o largo plazo, que posee una elevada significación emocional o sentido personal para el sujeto y de las estrategias correspondientes para el logro de los objetivos propuestos" (Humarán y Domínguez, 2014, pág. 88).

Humarán y Domínguez (2014), así mismo mencionan a Ovidio D' Angelo, al referirse a los proyectos de vida. "Estos proyectos constituyen una formación psicológica en la que se manifiestan las determinaciones sociohistóricas del sujeto, matizadas por su comprensión individual de la realidad objetiva. (...) Es importante, en la elaboración del proyecto de vida, el logro de una coherencia entre el uso del tiempo actual y los planes de empleo del tiempo futuro, así como su relación con las orientaciones valorativas e intereses del individuo. (...) Se establece a nivel de formaciones motivacionales complejas de la juventud, lo cual se expresa en otras formaciones, como los ideales y la concepción del mundo" (Humarán y Domínguez, 2014, pág. 87 y 88 ).

Uno de los pilares fundamentales para la acreditación y la recategorización de la UTEQ en la denominada "categoría B", fue precisamente el haber implementado de manera oportuna desde el año 2008, el Modelo Pedagógico por Competencias (MPC).

El Modelo Pedagógico por Competencias pretende una universidad científica, tecnológica y humanista, encaminada a la formación de profesionales integrales. Pero lo primero era entender: ¿Qué son las competencias?; ¿Qué caracteriza un proceso de formación por competencias? Las competencias, para Bravo y Sánchez, son un "conjunto de conocimientos, habilidades, actitudes y valores, combinados, coordinados e integrados en la acción, adquiridos a través de la experiencia, que permite al sujeto resolver problemas específicos, de forma creativa e independiente, en contextos singulares" (Bravo y Sánchez, 2008).

La formación de las competencias se construye en cada individuo, respondiendo a estructuras complejas diferenciables en cada persona, pero ésta, como ser social, las construye en su relación con los demás sujetos. Las competencias también tienen que ser aprehendidas. Nadie, por competente que llegue a ser, lo fue al nacer; sólo en la vida, en la sociedad, es posible la construcción de las competencias.

La competencia presupone un alto grado de dominio por parte del sujeto y versatilidad conceptual, explícita o implícita de un determinado campo de la cultura. Implica la puesta en acción de los contenidos básicos de dicho campo. Un proceso de selección de alternativas de actuación y toma de decisiones. La pertinencia de su aplicabilidad en un contexto determinado. Un nivel de alternativas en los resultados de su construcción y aprehensión. Haciendo que el sujeto protagonice su conocimiento.

En tal virtud, las competencias son la configuración didáctica que sintetiza la riqueza de la profesión y del profesional, siendo expresión totalizadora de las 
cualidades que debe poseer el egresado para su desempeño, en un contexto histórico-social determinado y concreto, permitiendo generar el proceso de desarrollo del microdiseño curricular de la Carrera (Ordaz, 2011).

Bravo y Sánchez sostenían que "si existiera un marco nacional de competencias en el país, las instituciones de Educación Superior debieran alinearse con ese esfuerzo nacional y usar las competencias y estándares del marco. De no existir un marco de competencias referenciales para las Carreras, se deben crear las normas o estándares educacionales provisorios de competencias, que constituyan las bases para los planes de estudio" (Bravo y Sánchez, 2008). Y fue precisamente en ese proceso histórico, que en el Ecuador se estructura y define la Ley Orgánica de Educación Superior. Ahí precisamente se aplica el principio de alineación a estándares nacionales y se justifica la aplicación del nuevo modelo de enseñanzaaprendizaje en la Universidad Técnica Estatal de Quevedo.

Los indicadores para el diseño curricular por competencias, es que éstas son el principio organizador de la formación, se describen en términos de resultados y la evaluación está centrada precisamente en la adquisición efectiva de dichas competencias. Por tanto, el aprendizaje está orientado hacia la práctica (Tejada y Arias, 2006).

La estructura organizativa del Modelo por Competencias en la Carrera, está integrada por tres elementos: la competencia, los módulos y las unidades de aprendizaje. Los módulos corresponderían a cada ciclo, semestre o período académico; integrado por un conjunto de unidades de aprendizaje, que nuevamente al 2015, se las denominó asignaturas.

Las competencias pretenden resolver un conjunto de problemas en la estructura del módulo. Para aquello, los módulos deben contar con: formas de organización, escenarios, objetivos, contenidos, métodos, recursos didácticos y evaluación. Todo esto se consolida y tributa, a través del denominado "Proyecto Integrador". Por su parte, la estructura de las Unidades de Aprendizaje se relaciona con: las competencias del módulo, las unidades de competencias, las unidades de aprendizaje que desembocan en tareas, los temas y los sistemas de clase. Estos últimos, deben tener: objetivos, contenidos, métodos, recursos, formas de organización y evaluación.

Estas acciones no se cumplen solas o porque están impresas en el papel del reglamento. Las hacen las personas, en el rol de docentes-tutores o de estudiantestutorados. La elaboración del Proyecto Integrador del Módulo, parte de un problema. Se generan tareas, que se consolidan en un proyecto integrador; que se elabora y presenta con las evidencias de desempeño; que debe ser defendido y que debe mostrar las evidencias del producto. Este proceso tiene una organización y debe ser evaluado en forma grupal e individual. Todo esto, con la guía pedagógica y metodológica del coordinador del proyecto integrador.

¿Pero qué ocurre cuando el docente tutor no ha entendido el modelo por competencias? ¿Qué pasa cuando el docente tutor no conoce aspectos de pedagogía y de metodología universitaria? ¿Cuáles son los resultados de la improvisación de tutores? ¿Quién regula la aplicación coherente de los lineamientos 
reglamentados del modelo por competencias? ¿Dicha regulación es eficiente? ¿Qué ocurre cuando el docente no pertenece a la Carrera y ni siquiera es docente del módulo en proceso? ¿Cuáles son las consecuencias de la mala dirección tutorial? ¿Cómo incide la personalidad del tutor en el éxito de la tarea lograda?

Pues bien, un factor fundamental para la ejecución eficaz del proceso, es la indisciplina. Muy pocos docentes-tutores, manejan disciplinada y honestamente el proceso. Los/las estudiantes han aplicado lo aprendido. Los datos de la encuesta universitaria sobre la que inicialmente se problematizó este trabajo, así lo determinan de manera muy generosa. La realidad es todavía más evidente: cubículos abandonados, ausencia de estudiantes a las tutorías, informes académicos mensuales sin sustentación real, actitud negligente de tutores y tutorados, acreditación hacia ciclos posteriores con carencias significativas en conocimientos y competencias de la profesión y metodológicas, trabajos clonados y sin implicación cooperativa del grupo, entre otros aspectos criticables.

Sin embargo, luego de diez años de aplicación del modelo por competencias, también se pueden visualizar los cambios ocurridos, cuando existieron tutores eficientes y estudiantes que se disciplinaron con el proceso. Son notorias las diferencias al momento de egresar. Hay más solvencia académica, capacidad metodológica más fortalecida, comunicación y disertación segura y asertiva, logro de competencias profesionales. Pero de ahí, a lograr una tutoría de calidad y de excelencia, precisa de nuevos aportes y de consolidar la estructura del modelo con absoluta disciplina.

\section{Métodos}

Este enfoque del fundamento del modelo educativo, fortalece la apreciación que los investigadores han tomado en relación con las normativas y leyes nacionales del Ecuador. La UTEQ afinó su gestión académica con sobrados méritos durante esta década. Sin embargo, la parte más frágil del proceso se encuentra en la docencia. En el papel que se atribuyen los profesionales-docentes universitarios, que asumen un rol que discrepa en la realidad con los verdaderos propósitos de la tarea. Es por eso que, sostenemos lo que el criterio de los/las estudiantes revela al respecto.

La iniciativa doctoral por investigar el tema tutorial universitario, continua su proceso, siendo que para el 2018, los avances que se realizaron han entrado en un proceso involutivo y en algunos casos en estancamiento, frente al desconocimiento original de la tarea de construcción integral de las competencias profesionales, donde el papel del docente-tutor constituye el eje fundamental de esta caracterización. Acotemos información a partir de algunos elementos cuestionados:

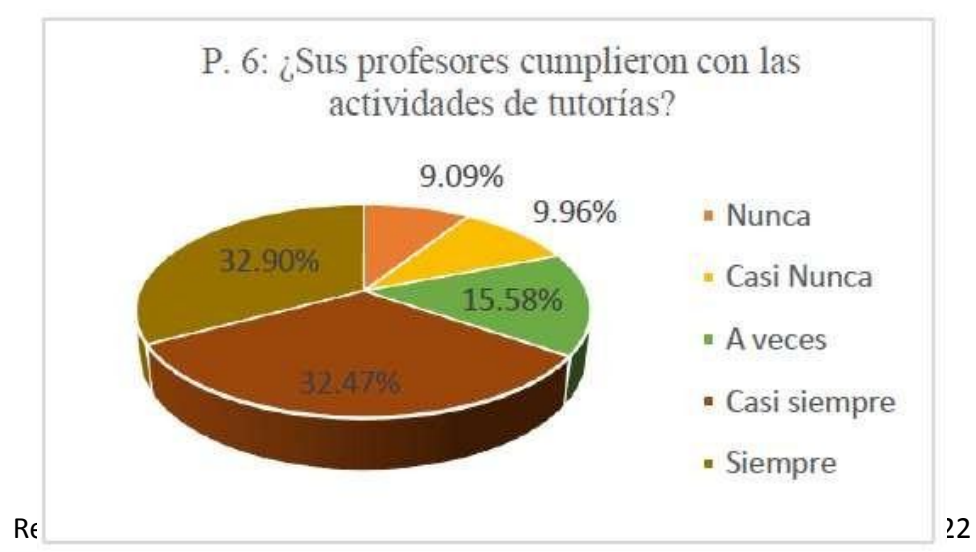




\section{Gráfico No. 1. Relacionado con la pregunta 6 de la investigación, respecto a si los profesores cumplieron con las actividades de tutorías \\ Fuente: Investigación a cargo del Eco. Daniel Parra Gavilanes año 2014}

Es notable el hecho de que apenas el 32,9\% de los docentes, cumplieron siempre con el proceso tutorial. La irregularidad y la significativa indiferencia de compromiso académico, refleja la evidente importancia de esta investigación relacionada con el claustro docente de la universidad ecuatoriana.

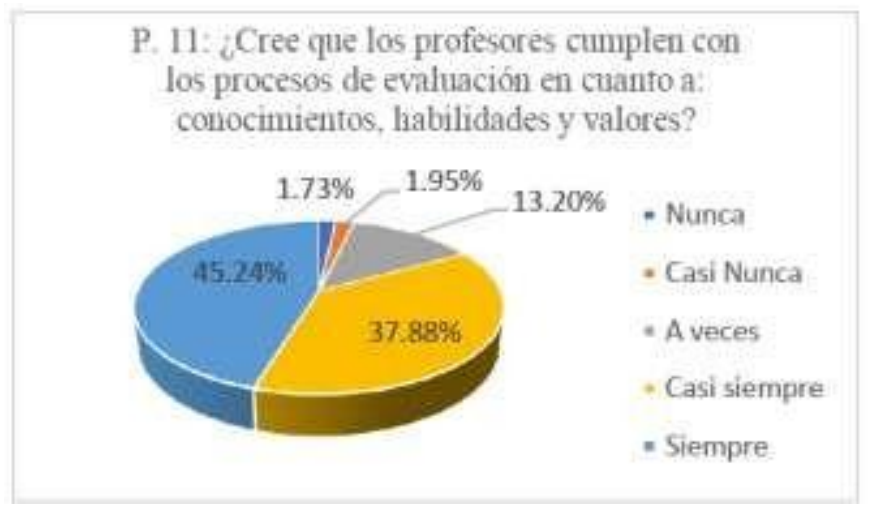

\section{Gráfico No. 2. Relacionado con la pregunta 11 de la investigación, respecto a si los profesores cumplen con los procesos de evaluación en cuanto a: conocimientos, habilidades y valores \\ Fuente: Investigación a cargo del Eco. Daniel Parra Gavilanes año 2014}

Un poco menos de la mitad de los docentes, el 45,24\%, siempre cumplen con los procesos de evaluación, en conocimientos, habilidades y valores. ¿Será que en este aspecto cuentan de manera importante, los conocimientos que sobre psicopedagogía y andragogía, deben tener como competencia los docentes? De otra manera, no se justifica el incumplimiento de exigencias significativas del proceso de enseñanza-aprendizaje.

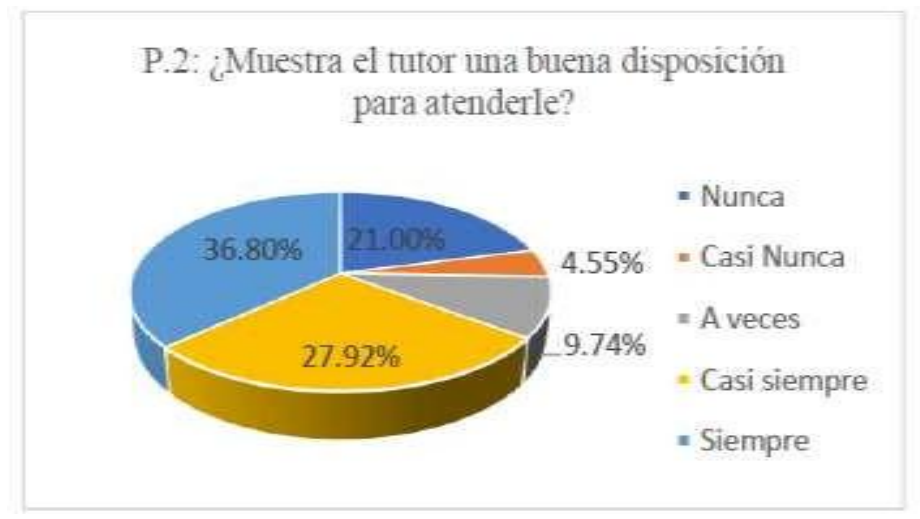




\section{Gráfico No. 3. Relacionado con la pregunta 2 de la investigación, respecto a si el tutor muestra una buena predisposición para atender al estudiante Fuente: Investigación a cargo del Eco. Daniel Parra Gavilanes año 2014}

La predisposición para intercambiar acciones tutoriales, refleja indudablemente el grato de empatía, compromiso axiológico, responsabilidad académica, interés laboral, del funcionario que cumple el rol de académico, frente al grupo de estudiantes que tiene a su cargo guiar, asesorar, conducir, tutorar. Apenas el $36,8 \%$ logra hacerlo siempre. Y sorprendentemente, el $21 \%$ nunca muestra esa predisposición. Lo que equivaldría a que uno de cada cinco profesores no es apto para la función que desempeña en la universidad.

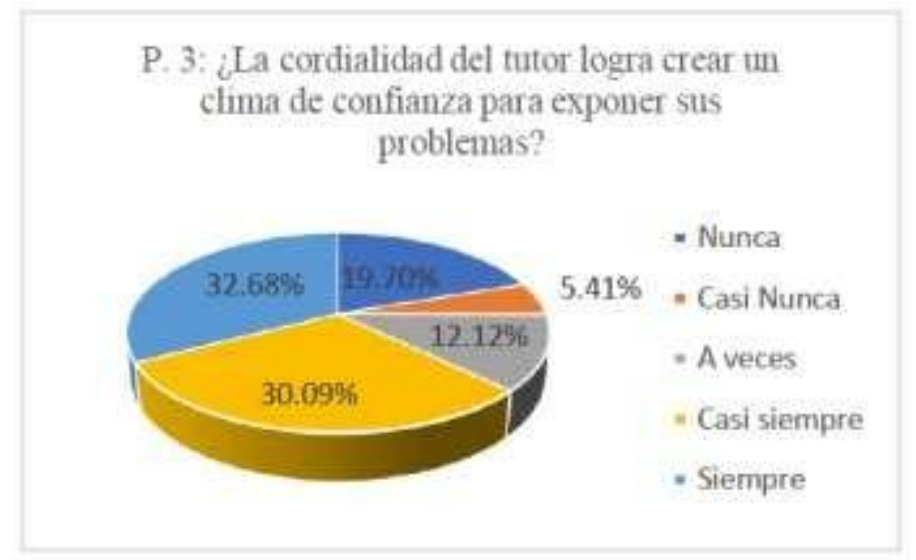

\section{Gráfico No. 4. Relacionado con la pregunta 3 de la investigación, respecto saber si el tutor muestra cordialidad que permita crear un ambiente de confianza para exponer sus problemas al estudiante}

Fuente: Investigación a cargo del Eco. Daniel Parra Gavilanes año 2014

Uno de los aspectos más criticables por parte de los estudiantes, es la carencia de habilidades sociales de comunicación y de criterios axiológicos que tienen los profesores. En muchos de los casos, generando discrepancias, extremados límites, obstáculos de vinculación académica, que alcanza incluso los niveles de acercamiento colaborativo y autónomo que debe desarrollar el/la estudiante. Restringidamente, el $32,68 \%$ de los profesores, si alcanza esas condiciones cordiales que permiten generar ambientes de confianza en la relación docenteestudiante.

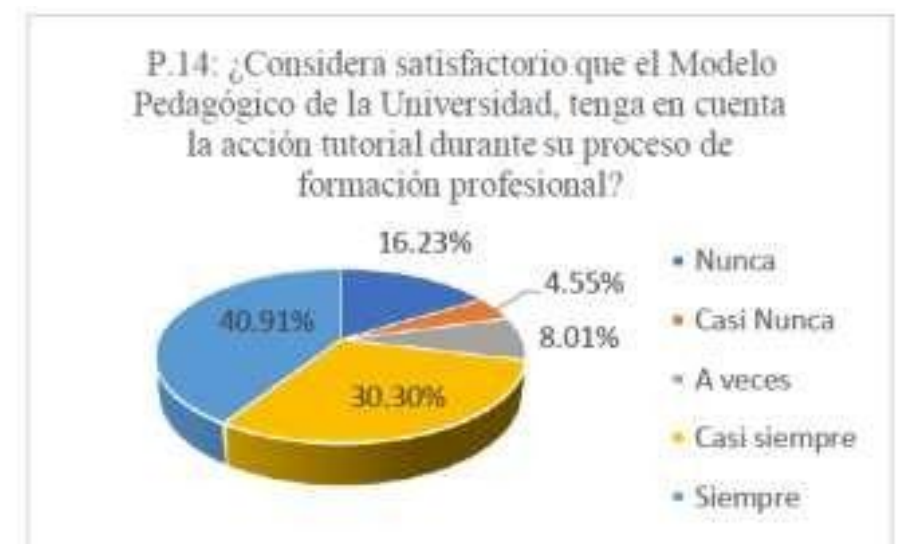




\section{Gráfico No. 5. Relacionado con la pregunta 14 de la investigación, respecto a si el estudiante considera satisfactorio que el MPC, tenga en cuenta la acción tutorial en el proceso de formación profesional \\ Fuente: Investigación a cargo del Eco. Daniel Parra Gavilanes año 2014}

Los/Las estudiantes denotan conocimiento y aceptación del modelo educativo de la Universidad, así como también la importancia de la acción tutorial que implica el proceso de formación profesional. De ahí, que cerca del $60 \%$ no vean con satisfacción los niveles de cumplimiento del rol tutorial del docente de la UTEQ.

\section{Conclusiones y discusión}

Esta tercera parte de la investigación en torno a la acción tutorial en la Universidad Técnica Estatal de Quevedo, muestra la importancia de entender el modelo educativo que cobija las acciones de la comunidad universitaria de la UTEQ, como se ha podido reflejar en los principales resultados obtenidos.

Los diez años de aplicación del modelo no han sido suficiente tiempo para cimentar las estrategias metodológicas de la construcción de competencias profesionales y humanas.

Hace falta principalmente, que las actividades propias del docente en su rol de tutor y guía, prevalezcan y adquieran una dimensión cada vez más estructurada y consecuente con las necesidades formativas integrales que tienen los/las estudiantes. Para esto, será imprescindible la preparación y la praxis que el docente universitario requiera implementar.

Generar convicción en relación al significado de este proceso psicopedagógico, requerirá una significativa y solvente motivación para mejorar las responsabilidades descritas por el modelo educativo.

\section{Referencias bibliográficas}

Bravo, G. y Sánchez, L. (2008). El profesor-tutor en el Modelo Pedagógico por competencias. Seminario en la Universidad Técnica Estatal de Quevedo. Quevedo: UTEQ.

González Maura, V. (2006). El profesor tutor: una necesidad de la universidad del siglo XXI. Revista Cubana de Educación Superior, Vol. 26 (2), pp. 23-36.

Horruitiner Silva, P. (2010). La universidad latinoamericana en la época actual. En: Cursos precongreso Universidad 2010. Ciudad de La Habana: Editorial Universitaria, 2010. - ISBN 978-959-16-1172-7-- 54 pág.

Humarán Barreiro, Y. y Domínguez García, L. (2014). Formación profesional y proyectos de vida de los estudiantes en la educación superior cubana. En C. d. Superior., Juventud y grupos en la educación superior. (pág. 230). La Habana: 
U H Facultad de Artes y Letras. Universidad de La Habana.

Ordaz Hernández, M. (2011). Concepción psicopedagógica del proceso de orientación: Estrategia para su implementación en la Universidad de Pinar del Río. Pinar del Río.

Tejada Tayabas, J. M. y Arias Galicia, L. F. (2006). El significado de tutoría académica en estudiantes de primer ingreso a licenciatura. En U. A. Potosí. San Luis de Potosí: División de Estudios de Posgrado, Facultad de Contaduría y Administración.

Travieso Valdés, D. y Figuerola Domenech, M. C. (2014). La educación superior cubana y el joven universitario: una polémica inconclusa. En C. d. (CEPES), Juventud y grupos en la educación superior (pág. 230). La Habana: UH Facultad de Artes y Letras, Universidad de La Habana.

UTEQ (2012). Reglamento para el Trabajo Académico y Metodológico del Modelo Pedagógico por Competencias (MPC). Quevedo: Universidad Técnica Estatal de Quevedo. 